\title{
EDITORIAL
}

\section{Current questions and controversies in chromosome fragile site research: does $W W O X$, the gene product of common fragile site FRA16D, have a passive or active role in cancer?}

Cell Death Discovery (2015) 1, 15040;

doi:10.1038/cddiscovery.2015.40; published online 19 October 2015

- The controversy about common fragile site (CFS) and their associated genes/products is whether their aberrations in cancer passively accumulate or actively contribute to cancer development.

- The WW domain-containing oxidoreductase (WWOX) gene spans one of the most active CFS FRA16D and is commonly altered in cancer.

- WWOX encodes a tumor suppressor that enhances efficient DNA damage response (DDR) functions ranging from DNA repair and apoptosis to suppression of tumorigenic signaling pathways.

- WWOX deficiency results in cancer progression not only because of the fragility but also because it is enriched by selective pressure during tumorigenesis.

The role of CFSs in cancer is debatable and two major views dominate the discussion in this regard. One view suggests that CFSs are hotspots for genomic instability leading to damage of genes residing within them and hence losing these genes is unselected 'passenger' or 'passive' event. The counter argument is that losing these genes leads to selective pressure as a result of their tumor-suppressive activities and therefore directly contributes to carcinogenesis and have an active role: a 'driver' event.

Genomic instability, one of cancer hallmarks, is characterized by mutations, deletions, amplifications and translocations. ${ }^{1}$ In particular, we focus here on genomic aberrations related to large deletions since CFSs have been known for decades as 'hotspots' for deletions and breaks taking place in metaphase chromosomes as a result of replication stress. ${ }^{2}$ Indeed, the most significant deletions in human tumor specimens and cancer cell lines coincide with CFSs or large genes. ${ }^{3-5}$ Many of these deletions are hemizygous, suggesting, although not proven, that the other allele is maintained. This assumption led to the hypothesis that alterations in CFSs are secondary events that do not contribute to the multistep tumorigenesis process. However, emerging evidence suggests elsewise as CFSs do not seem to be inert structures but contain functional elements, such as tumor suppressor genes, coding and noncoding, that have critical roles in carcinogenesis. ${ }^{6}$

One such example of a tumor suppressor gene is the WW domain-containing oxidoreductase (WWOX), which spans the CFS FRA16D. WWOX is among the most significantly deleted genes in cancers, ${ }^{3,4,7}$ suggesting a selective pressure because of a tumor suppressor activity and also because of the fragility of that locus. Accordingly, emerging findings demonstrate that WWOX protein has pleiotropic tumor-suppressive functions, particularly by regulating the DNA DDR. ${ }^{8}$

The WWOX/FRA16D locus, similar to other CFS genes, is an evolutionarily conserved, megabase long that is predisposed to DNA single- and double-strand breaks (SSBs and DSBs, respectively) in response to intrinsic (oncogenic) or extrinsic (environmental) replication stress. Therefore, WWOX and other CFS genes might be an early warning sensor for DNA damage. Because of their sensitivity, they are prone to allelic imbalances and deletions associated with genomic instability and mutator phenotype that further drive tumor progression. Here we discuss supporting evidence favoring active and tumor suppressor functions of WWOX in tumorigenesis.

\section{ALTERATIONS OF WWOX IN CANCERS SUPPORT ITS ROLE AS A TUMOR SUPPRESSOR}

WWOX was originally cloned as a putative tumor suppressor because of its frequent loss in cancer (reviewed in Gardenswartz and Aqeilan ${ }^{7}$ ). Data obtained from the TCGA copy number portal demonstrated that the WWOX locus is the fifth most frequently deleted locus in 10844 samples from 33 cancer types (Q-value = 1.31 $\mathrm{E}^{-266}$; http://www.broadinstitute.org/tcga/home). Well-known tumor suppressors such as PTEN, RB1 and TP53 are significantly deleted $\left(0,7.24 \mathrm{E}^{-273}\right.$ and $5.04 \mathrm{E}^{-22}$, respectively), although the deleted regions have additional genes within their peaks (i.e. PTEN with the tumor suppressor KLLN); WWOX, on the other hand, is a single gene within the deleted peak emphasizing that losing its own activity contributes to cancer. In addition, translocations within this region $(14 q 32 ; 16 q 23)$ were observed in up to $25 \%$ of multiple myelomas. ${ }^{7}$

Loss of WWOX is associated with poor prognosis in numerous cancers and may result not only from deletions and translocations but also from epigenetic silencing by DNA methylation or mutations within its promoter. ${ }^{7}$

Another interesting observation is that most of the deletions within WWOX are hemizygous rather than homozygous, suggesting haploinsufficiency. In support of this notion, Wwox heterozygous mice develop higher incidence of spontaneous tumors. ${ }^{9}$ Furthermore, McAvoy et $a f^{5}$ reported that there is no relationship between alteration of a given CFS and expression of its associated gene product in a given cancer, suggesting that these alterations are not merely responsible for dysregulation of WWOX protein expression. It is therefore possible that at early stages of tumorigenesis, breaks in FRA16D alert the DDR to warn against environmental or intrinsic cues. But is this the end of the story or that a following chapter do exist?

\section{WWOX FUNCTIONS AS A TUMOR SUPPRESSOR}

Regardless of WWOX localization in the genome and causes of its aberration in cancer, emerging findings indicate that WWOX tumor-suppressive functions range from involvement in DDR to modulation of tumorigenic signaling such as HIF1 $a$, TGF $\beta / \mathrm{SMAD}$ and WNT/ $\beta$ catenin (reviewed in Aqelian et al. ${ }^{10}$ ).

\section{WWOX FACILITATES DDR}

Although WWOX locus is vulnerable to DNA damage, levels of WWOX protein were recently shown to be induced following 


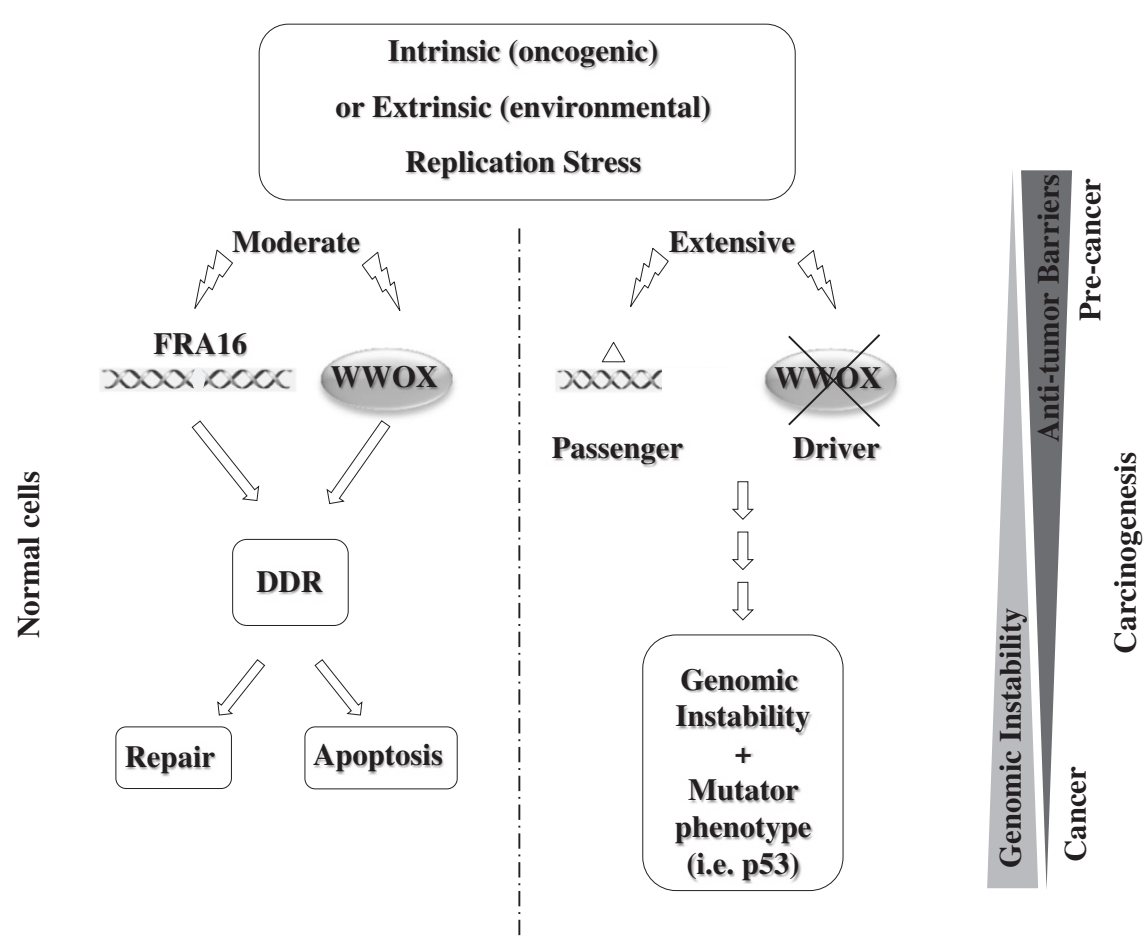

Figure 1. Hierarchical model for WWOX/FRA16D mediating cancer development. Both the fragile site FRA16D and its gene product WWOX are affected by replication stress. Although FRA16D is prone to DSBs, WWOX activity is induced resulting in DDR and/or apoptosis. Upon extensive damage, breaks within FRA16D inaccurately repaired as deletions. Cells with deletions within WWOX-encoding gene (depicted as $\Delta$ ) are positively selected owing to the role of WWOX in DDR leading to genomic instability and mutator phenotype. Additional mutations in other tumor suppressor loci (such as p53) would release antitumor barriers leading to cancer development.

DSBs ${ }^{8}$ and SSBs (unpublished data). Upon DSBs, WWOX physically interacts and supports activation of the checkpoint kinase ATM allowing efficient and accurate DNA repair. ${ }^{8}$ ATM is activated early after DSBs and phosphorylates the ubiquitin E3 ligase ITCH and other substrates, including $\mathrm{H} 2 \mathrm{AX}, \mathrm{CHK} 2$ and $\mathrm{p} 53 .^{8}$ ITCH mediates K63-linked ubiquitination of WWOX and promotes its translocation into the nucleus where it activates ATM in a positive feed-forward loop manner. ${ }^{8,10}$ WWOX deficiency results in reduced activation of ATM and defects in recruitment of components of the repair machinery to damaged sites, ${ }^{8}$ resulting in increased DSBs. Overall, loss of WWOX impaired DNA repair, which may lead to genomic instability and tumorigenesis. ${ }^{10}$

\section{WWOX PROMOTES APOPTOSIS}

Ectopic expression of WWOX in different cancer cell lines resulted in apoptosis in vitro and marked inhibition of tumorigenicity in vivo. Cumulative evidence has shown that WWOX regulates cell death through interactions with members of the p53 family of proapoptotic transcription factors p53, p73 and p63 through various mechanisms. Although the interaction between WWOX and p53 occurs only when phosphorylated WWOX enters the nucleus, ${ }^{11}$ cytoplasmic unphosphorylated WWOX can interact with p73 leading to transactivation-independent apoptosis. WWOX interaction with $\triangle \mathrm{Np} 63 \mathrm{a}$ inhibits its nuclear translocation and suppresses its transactivation function. ${ }^{12}$ In addition, WWOX has also been shown to interact with mTOR to suppress autophagy and induce apoptosis and methotrexate sensitivity. ${ }^{13}$

\section{CONCLUSIONS}

Tumor-suppressing functions of WWOX identify it as a master regulator that protects from genomic instability (but also having major roles in other cellular process that could not be discussed here). Therefore, we propose that WWOX loss early in the precancerous stage is not a secondary/passive event because of the fragility of its locus but provides a growth advantage that actively contributes to tumorigenesis. Further studies of WWOX function might shed light on the intriguing question of why one of the most unstable chromosomal regions in the genome, FRA16D, is evolutionarily conserved and harbors a tumor suppressor? One explanation might be that cancer usually occurs after the reproductive phase and therefore loss of CFS would not have an inherited selective pressure. In support of this assumption, germline mutations or loss of function of WWOX are associated with neuronal disorders, ${ }^{14}$ whereas somatic WWOX lesions are associated with cancer. ${ }^{15}$

Gorgoulis and co-workers ${ }^{6}$ suggested that scattering 'sensors' of coding and noncoding elements in CFSs throughout the genome send alarming signals of replication stress. We further propose that as part of the DDR, gene products of CFSs, such as WWOX, ${ }^{10}$ have active roles in repairing the damage or eliminating the 'bad' cells by apoptosis. However, when WWOX is lost, the antitumor barriers are gradually lost and aberrations in classical recessive genes accumulate to feed into carcinogenesis (Figure 1). Therefore, placing a guarding gene within CSF provides an advantage as long as the cellular checkpoints are not compromised, whereas failure in these checkpoints (i.e. by additional mutations in RB1 and p53) would lead to tumor initiation and/or progression. Other CFS genes such as FHIT, SPIDR, PARK2 and RORA have also roles in DDR, with some having established tumor suppressor function. ${ }^{5,16}$ As the expression of these CFSs and their associated genes is cell-type-specific, ${ }^{17}$ it is reasonable to assume that they vary in their behavior from one cancer type to another. 
Further delineation of WWOX tumor suppressor functions and other products of CFSs, both in vitro and using animal models, should contribute to better understanding of carcinogenesis. Mapping and timing of CFS genes' inactivation during the neoplastic process, of different tissues, will be crucial to best dissect their contributions and nail their functions.

\section{COMPETING INTERESTS}

The authors declare no conflict of interest.

I Hazan ${ }^{1}$ and RI Aqeilan ${ }^{1}$

${ }^{1}$ Lautenberg Center for Immunology and Cancer Research, IMRIC, Hebrew University-Hadassah Medical School, Jerusalem, Israel Correspondence: Rl Aqeilan (ramiaq@mail.huji.ac.il)

\section{REFERENCES}

1 Hanahan D, Weinberg RA. Cell 2011; 144: 646-674.

2 Durkin SG, Glover TW. Annu Rev Genet 2007; 41: 169-192.

3 Beroukhim R et al. Nature 2010; 463: 899-905.
4 Bignell GR et al. Nature 2010; 463: 893-898.

5 McAvoy et al. Cytogenet Genome Res 2007; 118: 260-269.

6 Georgakilas AG et al. Cell Mol Life Sci 2014; 71: 4519-4544.

7 Gardenswartz A, Aqeilan RI. Exp Biol Med 2014; 239: 253-263.

8 Abu-Odeh M et al. Proc Natl Acad Sci USA 2014; 111: E4716-E4725.

9 Aqeilan Rl et al. Proc Natl Acad Sci USA 2007; 104: 3949-3954.

10 Aqeilan Rl, Abu-Remaileh M, Abu-Odeh M. Cell Mol Life Sci 2014; 71: 4589-4599.

11 Chang et al. Trends Mol Med 2007; 13: 12-22.

12 Salah Z et al. Cell Death Dis 2013; 4: e480.

13 Tsai CW et al. Cell Death Dis 2013; 4: e792.

14 Tabarki B, Al Mutairi F, Al Hashem A. Exp Biol Med 2015; 240: 400-402.

15 Yang L et al. Hum Mol Genet 2013; 22: 1886-1894.

16 Waters CE et al. Cell Mol Life Sci 2014; 71: 4577-4587.

17 Le Tallec B et al. Cell Mol Life Sci 2014; 71: 4489-4494.

(i) This work is licensed under a Creative Commons Attribution 4.0 International License. The images or other third party material in this article are included in the article's Creative Commons license, unless indicated otherwise in the credit line; if the material is not included under the Creative Commons license, users will need to obtain permission from the license holder to reproduce the material. To view a copy of this license, visit http://creativecommons.org/licenses/ by/4.0/ 